\title{
Design of a Wedge Plate for Polarization-Preserving Angular Shifter
}

\author{
Yi-Kai CHENG* and Jyh-Long CHERN \\ Department of Photonics, Institute of Electro-Optical Engineering, Microelectronics and Information System Research Center, \\ National Chiao Tung University, Hsinchu 300, Taiwan
}

(Received July 16, 2008; Revised November 26, 2008; Accepted December 4, 2008)

\begin{abstract}
It is shown that by using a wedge plate, the incident direction of light propagation can be rotated as necessary while still preserving beam polarization. The basic conditions of these preservations of polarization are deduced. For numerical demonstration, it is shown that a collimated beam with $+45^{\circ}$ linear polarization can be guided to any pre-settled direction where the square of ellipse ratio variation in the polarization is less than $0.0001 \%$, while the variation of squared ellipse ratio of a right circular polarization beam is kept under $0.1 \%$. (C) 2009 The Optical Society of Japan
\end{abstract}

Keywords: wedge plate, polarization, optical designs

\section{Introduction}

In many practical applications in optics where manipulation of light is essential, preserving the polarization of a beam of light after propagation is critical and has been used in a variety of fields such as biological tissue light probes, or ellipsometers for refractive index, film thickness measurement, etc. ${ }^{1,2)}$ When light is propagated, displaced, or deflected, its polarization is usually changed. For example, when light bounces off a reflective mirror, the reflection introduces an additional phase that alters the polarization of the light beam. In the literature, Azzam suggested solutions to displace a monochromatic light beam parallel to itself without change of polarization by utilizing a pair of parallel mirrors with a single dielectric layer coating. ${ }^{3,4)}$ On the other hand, Cojocaru ${ }^{5)}$ and Wang et al. ${ }^{6)}$ proposed the designs of coating layers with totally reflecting prism to provide several convenient and efficient methods to control the phase retardance. Meanwhile, Galvez presented the combinations of four totally reflecting prisms to preserve polarization. ${ }^{7}$ ) Galvez' scheme has benefits in achromatic application and very good tolerance which can preserve the polarization with a square of ellipse ratio variation of less than $1 \%$ while one degree of component tilt occurs. ${ }^{8)}$ These early studies provide very useful schemes to maintain polarization, but they generally focus on the displacement of a light beam, i.e., when the propagating direction of a displaced beam is parallel to the original beam. However, angular shifting is generally employed to reduce the optical path of beam propagation and hence the system size, which has practical value. Technically, polarization-preserving angular shifting provides more flexibility in optical engineering applications, e.g., a polarized light probe with angular incident injection could provide another useful scheme in exploring 3D structure and response. However, it remains quite uncertain and difficult to deflect the beam direction while maintaining the state of polarization using this technique. Recently it has been shown that by using a wedge plate, it is possible to provide a different scheme of a polarization-preserving beam

\footnotetext{
*E-mail address: ykcheng.eo91g@nctu.edu.tw
}

angular shifter. ${ }^{9)}$ A polarization-preserving wedge has an advantage in manufacture and has better extendibility on the applications of polarization controlling mechanisms while the plate provides both spatial and angular shift of the beam propagation path; and furthermore, a zero vertex angle can reduce a wedge angular shifter to become a beam displacer.

However, we had shown in ref. 9 the deduction of polarization-preserving issue of the wedge plate was still limited to the cases of even-time light bounce (reflection within wedge plate) and linearly polarized incident light. Hence, it is worthwhile to evaluate the situation of oddnumber reflection as well as the other case of polarization, e.g., circular polarization. On the other hand, the variation of index of refraction may be crucial in practical applications and hence, the investigation of tolerance on this factor is also provided.

This paper is organized as follows. Section 2 provides basic formalism of polarized ray tracing in dielectric-filled material. Section 3 presents the issue of polarization conservation and its conditions. Section 4 shows the simulation verification. Section 5 investigates the tolerance issue of manufacturing accuracy and incident beam angle and refractive index, and the final section contains conclusions.

\section{Basic Principle: Propagation Formalism of Polarized Light with the Muller Matrix}

For completeness, let us first summarize the general properties of a polarized ray propagating in a dielectric-filled material. According to Fresnel's equations, the transmitted and reflected fields of a light beam on an air-dielectric interface follows: ${ }^{10)}$

$$
\begin{aligned}
R_{s} & =\frac{\cos \theta_{i}-n \cos \theta_{t}}{\cos \theta_{i}+n \cos \theta_{t}} E_{s}, \\
R_{p} & =\frac{n \cos \theta_{i}-\cos \theta_{t}}{n \cos \theta_{i}+\cos \theta_{t}} E_{p}, \\
T_{s} & =\frac{2 \cos \theta_{i}}{\cos \theta_{i}+n \cos \theta_{t}} E_{s}, \\
T_{p} & =\frac{2 \cos \theta_{i}}{n \cos \theta_{i}+\cos \theta_{t}} E_{p} .
\end{aligned}
$$


In eq. (1), $E, R$, and $T$ represent the incident, reflected, and refracted fields, and the subscripts $s$ and $p$ denote the parallel and perpendicular directions, respectively. $\theta_{i}$ is the incident angle, $\theta_{t}$ is the angle of refraction, and $n$ is the refractive index of the dielectric medium.

$$
M_{T}=\frac{\sin 2 \theta_{i} \sin \theta_{t}}{2\left(\sin \Theta_{+} \cos \Theta_{-}\right)^{2}}\left(\begin{array}{c}
\cos ^{2} \Theta_{-}+1 \\
\cos ^{2} \Theta_{-}-1 \\
0 \\
0
\end{array}\right.
$$

where $\Theta_{ \pm}=\theta_{i} \pm \theta_{t}$, and for total internal reflection it follows that

$$
M_{T I R}=\left(\begin{array}{cccc}
1 & 0 & 0 & 0 \\
0 & 1 & 0 & 0 \\
0 & 0 & \cos \Delta & -\sin \Delta \\
0 & 0 & \sin \Delta & \cos \Delta
\end{array}\right),
$$

where

$$
\Delta=-2 \tan ^{-1}\left(\frac{\cos \theta_{i} \sqrt{n^{2} \sin ^{2} \theta_{i}-1}}{n \sin ^{2} \theta_{i}}\right) .
$$

Equations (2) and (3) govern the variations of polarization as a light ray interacts within a dielectric-filled material.

\section{Design Rules}

The key concern is: How can we design an optical component that is capable of guiding a ray to a special angular direction while simultaneously preserving its polarization? Technically, the total phase delay (or phase shift) during ray propagation must be kept as $2 \pi$, or an integral multiple of $2 \pi$, and the deflected angle between the exit and incident beams should match the designed value. It has been shown that a wedge plate is a possible candidate to achieve this goal. ${ }^{9)}$

Referring to ref. 9, and much like the wedge plates considered in refs. 12 and 13, Fig. 1(a) shows that a ray is incident to the narrow side of the wedge plate as long as the incident angle when the ray first strikes the top wedge surface is greater than the total internal reflection angle $\theta_{c}$. This ray can continuously propagate between the top and bottom surfaces of the wedge plate until it reaches the wide side (or the exit surface). To avoid unnecessary complexity, the wedge input and exit surfaces were cut such that their directions of surface normal are the same as the directions of incident and exit beams, respectively.

In Fig. 1(a), $\alpha_{i}$ is the angle between the ray propagation direction and the horizontal axis after the $i$ th total internal reflection, $\theta_{i}$ is the incident angle before the $i$ th total internal reflection, $\theta_{v}$ is the wedge vertex angle, and $n$ is the refractive index of the plate material. Before leaving the wedge plate, this ray encounters $m$ times of total internal reflection. Figure 1(a) shows that one ray finally escapes from the top surface of the wedge plate and $m$ is an even
The Stokes parameter provides the formulism of the Mueller matrix for the reflection and refraction on an airdielectric interface from eq. (1). Referring to ref. 11, the Mueller matrix of transmission on the air-dielectric interface is

$$
\left.\begin{array}{ccc}
\cos ^{2} \Theta_{-}-1 & 0 & 0 \\
\cos ^{2} \Theta_{-}+1 & 0 & 0 \\
0 & 2 \cos \Theta_{-} & 0 \\
0 & 0 & 2 \cos \Theta_{-}
\end{array}\right)
$$
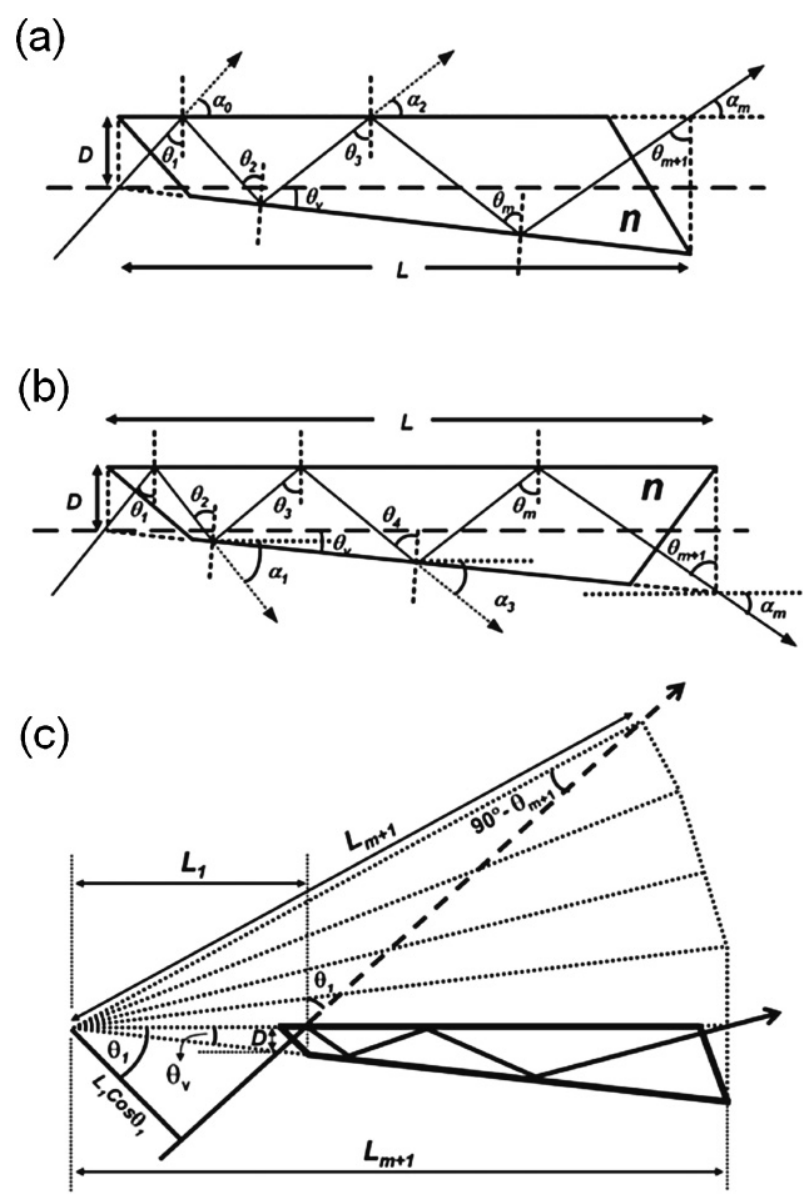

Fig. 1. Schematic diagrams of ray propagating where (a) wedge plate and (b) a virtually folded wedge plate.

number. However, another possibility will also come up. Figure 1(b) shows that ray incidents in to the wedge and escapes from the bottom surface of the wedge plate. In this case, this ray will encounter odd times of total internal reflection during the propagation. The geometry in Figs. 1(a) and 1(b) shows that $\theta_{i}=\theta_{1}+(i-1) \theta_{v}, \alpha_{i}=90-\theta_{i}-\theta_{v}$ (when $i$ is even) or $\alpha_{i}=-\left(90-\theta_{i}\right)$ (when $i$ is odd), and the ray deflection angle for $\mathrm{m}$ times of reflection is $\Delta \alpha=$ $\alpha_{0}-\alpha_{m}=m \theta_{v}$ (when $i$ is even) or $\Delta \alpha=2 \alpha_{0}-m \theta_{v}$ (when $i$ is odd). In short, 
(a)

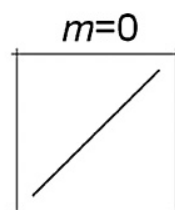

$S_{0}=1$

$S_{1}=0$

$S_{2}=1$

$S_{3}=0$

(b)

$e^{2}=0$

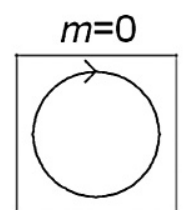

$S_{0}=1$

$S_{1}=0$

$S_{2}=0$

$S_{3}=1$

$e^{2}=1$

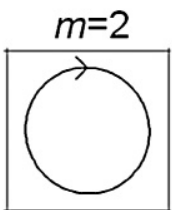

$S_{0}=0.9581$

$S_{1}=0$

$S_{2}=0.0232$

$S_{3}=0.9578$

$e^{2}=0.9523$

$m=2$

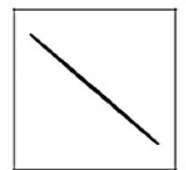

$S_{0}=0.9581$

$S_{1}=0$

$S_{2}=-0.9577$

$S_{3}=-0.0233$

$e^{2}=1.4769 \times 10^{-4} \quad e^{2}=0.6649$

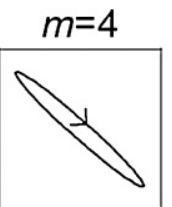

$S_{0}=0.9581$

$S_{1}=0^{6}$

$S_{2}=-0.9384$

$S_{3}=-0.1928$

$e^{2}=0.0103$

$m=4$

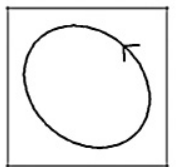

$S_{0}=0.9581$

$S_{1}=0$

$S_{2}=-0.1928$

$S_{3}=-0.9385$

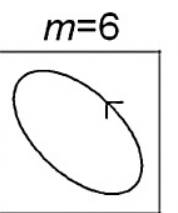

$S_{0}=0.9581$

$S_{1}=0$

$S_{2}=-0.5262$

$S_{3}=-0.8006$

$e^{2}=0.2909$

$m=6$

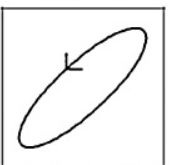

$S_{0}=0.9581$

$S_{1}=0$

$S_{2}=-0.8001$

$S_{3}=-0.5262$

$e^{2}=0.0895$

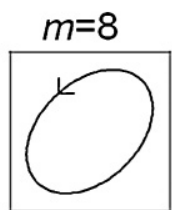

$S_{0}=0.9581$

$S_{1}=0$

$S_{2}=0.3581$

$S_{3}=-0.8886$

$e^{2}=0.4559$

$m=8$

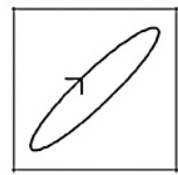

$S_{0}=0.9581$

$S_{1}=0$

$S_{2}=-0.8886$

$S_{3}=0.3581$

$e^{2}=0.0376$

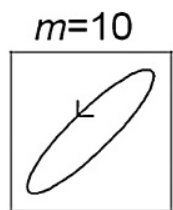

$S_{0}=0.9581$

$S_{1}=0$

$S_{2}=0.8554$

$S_{3}=-0.4313$

$e^{2}=0.0566$

$m=10$

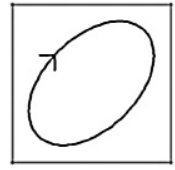

$S_{0}=0.9581$

$S_{1}=0$

$S_{2}=0.4343$

$S_{3}=0.8554$

$e^{2}=0.3791$

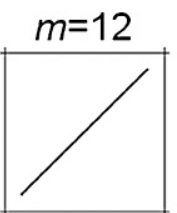

$S_{0}=0.9142$

$S_{1}=0$

$S_{2}=0.9142$

$S_{3}=-1.0783 \times 10^{-4}$

$e^{2}=3.1671 \times 10^{-9}$

$m=12$

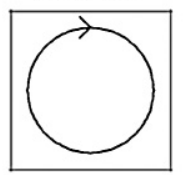

$S_{0}=0.9142$

$s_{1}=0$

$S_{2}=-1.0783 \times 10^{-4}$

$S_{3}=0.9142$

$e^{2}=1-2.2598 \times 10^{-4}$

Fig. 2. Polarization variations when total internal reflections happen with different incident beams, (a) $+45^{\circ}$ linearly polarized beam and (b) right circular polarized beam.

$$
\left\{\begin{array}{ll}
\Delta \alpha= \begin{cases}m \theta_{v} & (\text { when } m \text { is even }) \\
\pi-2 \theta_{1}-(m-1) \theta_{v} & (\text { when } m \text { is odd })\end{cases} \\
\sum_{i=1}^{m}-2 \tan ^{-1}\left(\frac{\cos \left[\theta_{1}+(i-1) \theta_{v}\right] \sqrt{n^{2} \sin ^{2}\left[\theta_{1}+(i-1) \theta_{v}\right]-1}}{n \sin ^{2}\left[\theta_{1}+(i-1) \theta_{v}\right]}\right)=-2 \pi \cdot k
\end{array},\right.
$$

where $m$ and $k$ are integers. After specifying the ray deflection angle $\Delta \alpha$, multiple solutions of $\left(\theta_{1}, \theta_{v}\right)$ can be derived from eq. (4) with different values of $m$ and $k$. Equation (4) is the basic condition of a polarizationpreserving angular shifter for a wedge plate. Formally, $\theta_{1}$ and $\theta_{v}$ are the dominant parameters in eq. (4), but the real scale of the wedge plate, i.e., the width of input port $D$, and the total length of wedge $L$, provide the degree of freedom in an angular shifter.

\section{Numerical Exploration}

For a numerical illustration, consider a typical glass, BK7, where $n=1.51509$ at $632.8 \mathrm{~nm}$, and request $\Delta \alpha=30^{\circ}$. After solving, one solution was arbitrarily selected for demonstrations: $m=12, \theta_{1}=54.79^{\circ}, \theta_{v}=2.5^{\circ}$, where the total phase delay is $\sim-2 \pi$, i.e., $-2 \pi-1.13 \times 10^{-4}$. Figure 1(c) shows the series of a virtually folded wedge by which the multi-reflection ray path can be treated as a straight line. In Fig. 1(c), $L_{1}$ is the length from wedge vertex to first total internal reflection position and $L_{m+1}$ is the length from wedge vertex to $(m+1)$ th total internal reflection position. Simple mathematics prove that

$$
L_{1}=D\left(\cot \theta_{v}+\tan \theta_{1}\right)
$$

and

$$
L_{m+1}=\frac{L_{1} \cos \theta_{1}}{\sin \left(90^{\circ}-\theta_{m+1}\right)},
$$

and hence, the total length $L$ from the ray incident position to the exit position is

$$
\begin{aligned}
L & =L_{m+1}-L_{1}+D \tan \theta_{1} \\
& =D\left(\cot \theta_{v}+\tan \theta_{1}\right) \frac{\cos \theta_{1}}{\cos \left(\theta_{1}+m \theta v\right)}-D \cot \theta_{v} .
\end{aligned}
$$

By eq. (5), one can determine the exact size of the wedge plate by choosing the port width $D$ or the wedge length $L$.

In the numerical example above, $\theta_{v}=2.5^{\circ}$, and the thickness of the entrance port $D=3 \mathrm{~mm}$. By eq. (5), the plate length $L$ is $394.5 \mathrm{~mm}$. For simplicity of presentation (without loss of generality), ignore the absorption for the time being. We used TracePro (version 4.1.5), a commercial simulation package, for numerical verification. ${ }^{14)}$ A collimated beam with $+45^{\circ}$ linear polarization was propagated into the wedge plate at $\alpha_{0}=35.21^{\circ}$, and finally exited the wedge plate at $\alpha_{10}=5.21^{\circ}$ after 12 total internal reflections. Figure 2(a) shows the polarization states (the Stokes parameters) after the ray encountered even times of total internal reflection. Ellipse ratio $e$ of a linear polarized beam was considered and $e^{2}=I_{\min } / I_{\max }$ was calculated, where $I_{\max }$ and $I_{\min }$ are the maximum and minimum intensities of 
the optical beam, proportional to the squares of semimajor and semiminor axes of the ellipse, respectively, described by the beam's electric field vector. Results show that the output polarization beam has a good linear property, with the square of ellipse ratio $e^{2}=1.3332 \times 10^{-7}$.

Since in many applications, a circular polarized incident beam is considered, we also evaluate the propagation and polarization change. Figure 2(b) shows the simulation result with a right circular polarized beam, as an example, in which it is clearly indicated that the output beam also retains a very good circular polarization characteristic of a squared ellipse ratio $e^{2}=(1-2.2598) \times 10^{-4}$.

\section{Tolerance Issue}

A polarization-preserving wedge plate can be manufactured using glass molding or plastic injection. The accuracy of the incident plane angle and incident beam angle plays an important role in preserving the polarization state. Figure 1(a) already shows that the polarized beam normally incidents to the obliquely-cut input surfaces and exits from the output surfaces after $m$ total reflections. Incident angle errors negatively affect polarization-preserving shifter performance because there is additional phase delay.

This paper discusses three kinds of variation on tolerance when the ray is propagating.

(1) If the ray normally incident to the input surface which is tilted by a small angle $\delta_{s}$, i.e., the incident angle $\alpha_{0}{ }^{\prime}=\alpha_{0}+\delta_{s}$, then the ray angle after the $m$ th reflection is $\alpha_{m}{ }^{\prime}=\alpha_{0}+\delta_{s}+m \theta_{v}$ and the exit angle of the ray becomes $\alpha_{\text {out }}=\alpha_{0}+\sin ^{-1}(n \sin \delta)+m \theta_{v}=\alpha_{m}+\sin ^{-1}\left(n \sin \delta_{s}\right)$. The deflection angle of the output ray can be altered by another small angle $\sin ^{-1}\left(n \sin \delta_{s}\right)-\delta_{s}$, i.e., $\Delta \alpha^{\prime}=\Delta \alpha+$ $\sin ^{-1}\left(n \sin \delta_{s}\right)-\delta_{s}=m \theta_{v}+\sin ^{-1}\left(n \sin \delta_{s}\right)-\delta_{s}$.

(2) Even if the surfaces of the polarization-preserving wedge are manufactured identically, the misalignment of the incident beam with the incident plane still decreases the performance. When the incident beam is tilted by a small angle $\delta_{i}$, i.e., the incident angle $\alpha_{i n}=\alpha_{0}+\delta_{i}$, the ray angle before first reflection should be adjusted to $\alpha_{0}{ }^{\prime}=\alpha_{0}+$ $\sin ^{-1}\left(\sin \delta_{i} / n\right)$. The ray angle after the $m$ th reflection becomes $\alpha_{m}{ }^{\prime}=\alpha_{0}+\sin ^{-1}\left(\sin \delta_{i} / n\right)+m \theta_{v}$, and the ray exit angle finally could be $\alpha_{\text {out }}=\alpha_{0}+\delta_{i}+m \theta_{v}=\alpha_{m}+\delta_{i}$. Thus, an angular error in the output beam is the same as the incident beam's angular error. In other words, the ray deflection angle $\Delta \alpha$ remains unchanged when the incident beam is misaligned.

(3) When there is some variation on refractive index, or wavelength shift, an issue of tolerance has to be considered. Assume the wedge fabrication and incident beam alignment are perfect, the direction of the ouput beam will remail the same when refractive index varies, and this is due to the beam being still normalyl incident into wedge.

Angular errors on wedge surfaces or incident beam alignment do not seriously affect the deflection angle (or output ray direction), but the variation in the output beam's polarization state is unavoidable. The phase shift contributed by input and output surfaces, as deduced in eq. (2), must be considered as well. Figures 3(a) and 3(b) show the variations (a)

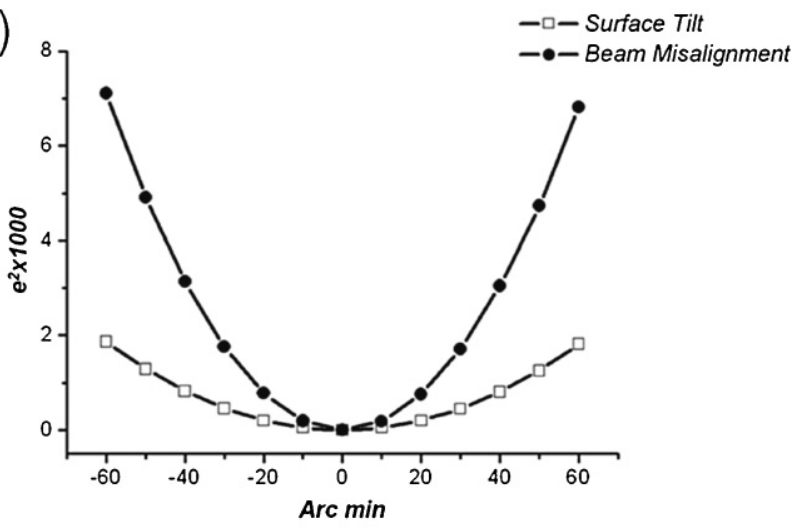

(b)

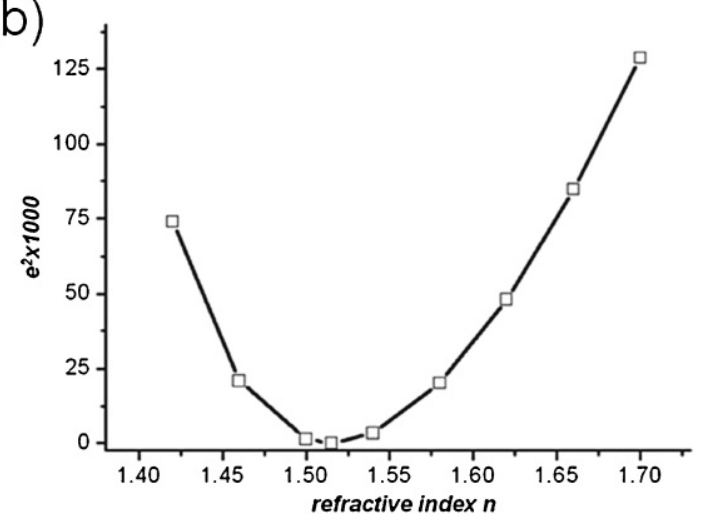

Fig. 3. Performance degradation caused by (a) incident surface tilt and incident-beam misalignment; (b) variation of refractive index.

of the square of ellipse ratio $e^{2}$ for the case of section 4 , where tolerance of input surface, incident beam alignment, and refractive index are considered. The incident beam carries a $+45^{\circ}$ linear polarization. The simulation result in Fig. 3(a) shows that variations of $e^{2}$ caused by one degree of the input surface error (or incident beam misalignment) are less than $1 \%$. On the other hand, Fig. 3(b) shows the impact of variation of refractive index which ranges from 1.42 to 1.7. As seen from the plot, the impact of the change of refractive index of 0.1 is to increase the variations of $e^{2}$ to $8 \%$.

\section{Conclusions}

In conclusion, a wedge plate is further shown able to angularly shift a collimated beam while maintaining its polarization state. The conditions for polarization preservation of both even and odd-number reflections are deduced and hence it provides the rules to design and manufacture a polarization-preserving angular shifter. More numerical examples, including circular polarization, have also been shown here. By the tolerance analysis, we could conclude that the incident beam angle's angular error or the variation of refractive index does not affect the deflection angle and the squared ellipse ratio can be kept under $1 \%$ with 1 degree accuracy for the incident beam angle. For the materials tolerance issue, it has also been shown that the 
error is kept under $8 \%$ with 0.1 accuracy of the refractive index.

Physically, the limit of the ray deflection angle $\Delta \alpha$ should be less than the incident angle $\alpha_{0}$ (for even reflections) or $2 \alpha_{0}$ (for odd reflections), while $\alpha_{0}$ should be less than $90^{\circ}-\theta_{c}$. For BK7 at a $632.8 \mathrm{~nm}$ wavelength, the critical angle $\theta_{c}$ is $41.3^{\circ}$; hence, the deflection angle $\Delta \alpha$ cannot be greater than $97.4^{\circ}$ when the wedge plate has an even number of reflections. Nevertheless, utilizing multiple polarizationpreserving wedges can increase the deflection angle. Polarized beams are widely used in measuring thin film, biological tissue, glass surface stress, and so forth. This study helps the designers to appreciate the ray polarization behavior within dielectric-fill material and to control the phase difference by properly choosing wedge parameters. The method shown here can easily be extended to other applications of polarization controlling mechanisms, for example, to replace the requirement of $2 \pi$ total phase with another value to create a defecting phase retarder.

\section{Acknowledgements}

This work was supported by the National Science Council of Taiwan, R.O.C., under contract no. NSC 96-2628-E-009-019-MY3. We thank the Lambda Research Corp. for the educational support of the simulation package, TracePro.

\section{References}

1) M. L. Faupel, S. B. Bambot, T. Harrell, and A. Agrawal: U.S. Patent 6975899 (2005).

2) R. M. Azzam and N. M. Bashara: Ellipsometry and Polarized Light (North-Holland, Amsterdam, 1989).

3) R. M. Azzam: Opt. Lett. 7 (1982) 80.

4) R. M. Azzam and M. Emdadur Rahman Khan: Appl. Opt. 21 (1982) 3314.

5) E. Cojocaru: Appl. Opt. 31 (1992) 4340.

6) Z. P. Wang, W. M. Sun, S. L. Ruan, C. Kang, Z. J. Huang, and S. Q. Zhang: Appl. Opt. 36 (1997) 2802.

7) E. J. Galvez: Opt. Lett. 26 (2001) 971.

8) A.-C. Hsu, C.-F. Ho, and J.-L. Chern: Appl. Opt. 41 (2002) 5956.

9) Y.-K. Cheng and J.-L. Chern: J. Opt. Soc. Am. A 25 (2008) 1558. The paper was accepted after this submission to ODF presentation.

10) M. Born and E. Wolf: Principles of Optics (Cambridge University Press, New York, 1999) 7th ed., pp. 42-43 and $50-51$.

11) E. Collett: Polarized Light: Fundamentals and Applications (Marcel Dekker, New York, 1992) pp. 139-161.

12) Y.-K. Cheng, S.-N. Chung, and J.-L. Chern: J. Soc. Inf. Disp. 14 (2006) 813.

13) Y.-K. Cheng, S.-N. Chung, and J.-L. Chern: J. Opt. Soc. Am. A 24 (2007) 2357.

14) See http://lambdares.com for technical information. 